\title{
A NEWBORN WITH ESOPHAGEAL ATRESIA, TRACHEOESOPHAGEAL FISTULA AND FEEDING PROBLEMS
}

\author{
Arda Ulaş Mutlu ${ }^{1}$ Oğuz Kızılkaya ${ }^{2}$, Mustafa İnan ${ }^{2}$ \\ ${ }^{1}$ Trakya University School of Medicine, Edirne, TURKEY \\ ${ }^{2}$ Department of Pediatric Surgery, Trakya University School of Medicine, Edirne, TURKEY
}

\begin{abstract}
Aims: Esophageal atresia is the most common congenital malformation of the esophagus. It can be diagnosed in the prenatal period, during the delivery or at the neonatal intensive care unit. With the operation, the respiratory system and digestive tract are being corrected to the anatomic position. In this case, we wanted to emphasize that patients with esophageal atresia may continue to have functional problems even after successful surgical treatment. Case Report: After preterm delivery, a female patient was consulted to Trakya University Department of Pediatric Surgery, at one day of age with the symptom of regurgitation of saliva. There were no abnormalities on physical examination. Due to the inability to pass an orogastric tube to the stomach, esophageal atresia was suspected. Thus, radiocontrast $\mathrm{x}$-ray study was performed: the proximal esophageal pouch was identified, and malformation was diagnosed. After the diagnosis, tracheoesophageal fistula has been ligated. The patient received physical therapy after the operation, and she was followed-up for 10 months. A full recovery was observed, and the patient was able to swallow food. Conclusion: Esophageal atresia with distal tracheoesophageal fistula is not an uncommon malformation. The patients can have problems with swallowing in their infancy even they are treated surgically in the neonatal period.
\end{abstract}

Keywords: Esophageal atresia, tracheoesophageal fistula, newborn

\section{INTRODUCTION}

Esophageal atresia (EA) consists of a discontinuity or atresia of the esophagus; with the majority of newborns exhibiting a connection or fistula between the esophagus and trachea (1). EA is seen in 1.9/10000 births and this frequency makes EA the most seen congenital malformation of the esophagus (1-3). Esophageal atresia could occur with the tracheoesophageal fistula (TEF) or without TEF. This congenital malformation could appear in different structures. Even they are all defined as EA, their differences made classification requisite. According to Gross (4), five sorts of EA have recognized (type A, B, C, D and E) being: type A is EA without TEF, type $B$ is proximal TEF with distal EA, type $C$ distal TEF with proximal EA, type D proximal and distal TEF and type $\mathrm{E}$ is TEF without EA, otherwise called " $\mathrm{H}$-type TEF". The most noted type is type C EA, being $87.5 \%$ of all cases (5).
The embryology of this malformation is not still fully defined (6). A newborn with this defect can have problems with breathing and swallowing. A meta-analysis made by Connor et al. (7) found out that the estimated prevalence of dysphagia between people with EA is $50.3 \%$. Today, EA with or without TEF can only be repaired surgically. This operation damages the nerves responsible for the esophageal motility. Dysmotility of the esophagus that leads to more problems like dysphagia, malnutrition, gastroesophageal reflux (GER) and complications arising out of prementioned problems (8).

Patients who have type C TEF can present more serious problems with breathing since regurgitation of gastric content, especially hydrochloric acid, damages the lungs and respiratory system irreversibly and cause lifelong continued breathing problems (8). Here we present the diagnosis and treatment of a patient with esophageal atresia and distal tracheoesophageal fistula.

Address for Correspondence: Arda Ulaş Mutlu, Trakya University School of Medicine, Edirne, TURKEY 


\section{CASE REPORT}

One day old patient consulted to Trakya University Department of Pediatric Surgery with the symptom of regurgitation of saliva after preterm delivery. She was $32+3$ week-gestation infant born by uneventful caesarian section. The birth weight was 1635 grams and polyhydramnios was noted during gestation. There were no abnormalities on physical examination and patient's Apgar score was 10 at the delivery. Due to the inability to pass an orogastric tube to the stomach, esophageal atresia was suspected. Thus, radiocontrast X-Ray study was performed, a proximal esophageal pouch was identified, and malformation was diagnosed (Figure 1). Echocardiography was performed for the cardiac examination, and the presence of right arcus aorta was reported. It is important for the strategy of surgical intervention. At the operation, the left thoracotomy was performed, and the tracheoesophageal fistula was repaired (Figure 2). Then, proximal and distal ends of the esophagus were primarily anastomosed over $10 \mathrm{Fr}$ nasogastric tube with 6/0 Polydioxanone absorbable sutures and thorax drain placed to the left thorax. At postoperative $2^{\text {nd }}$ day, the patient was fed with breastmilk from a nasogastric tube and on the $5^{\text {th }}$ day methylene blue was given orally to control the presence of anastomotic leakage. Thorax tube was removed on the $8^{\text {th }}$ day. However, nasogastric tube was not removed because the patient did not have an effective swallowing function. No postoperative complications were noted, and the patient was discharged with a nasogastric tube to be fed with. In the postoperative $2^{\text {nd }}$ month, radiocontrast video-fluoroscopy was performed, and no anastomotic stenosis or leakage was observed (Figure 3 ). In postoperative $8^{\text {th }}$ month, the patient was being fed from the feeding tube since the swallowing function was inadequate and there were no extra complications noted. In postoperative $10^{\text {th }}$ month, the patient's nasogastric tube was removed. She was referred to a physical medicine and rehabilitation specialist for chewing rehabilitation for a month. Following the rehabilitation, she was able to swallow food.

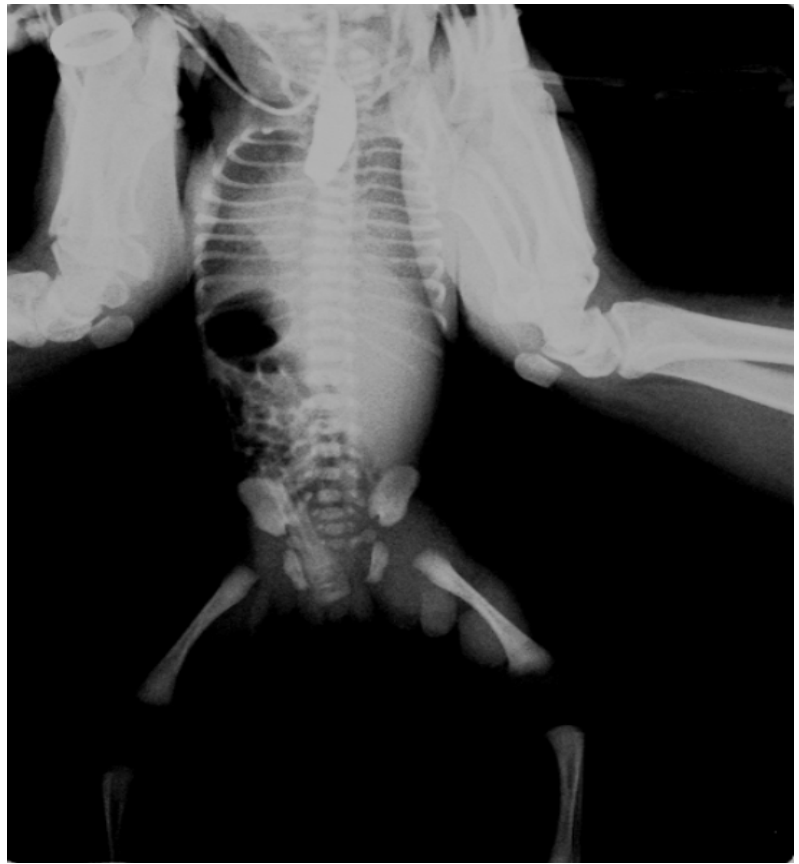

Figure 1: Preoperative plain X-Ray film (Radiocontrast liquid accumulated in proximal atresia is seen).

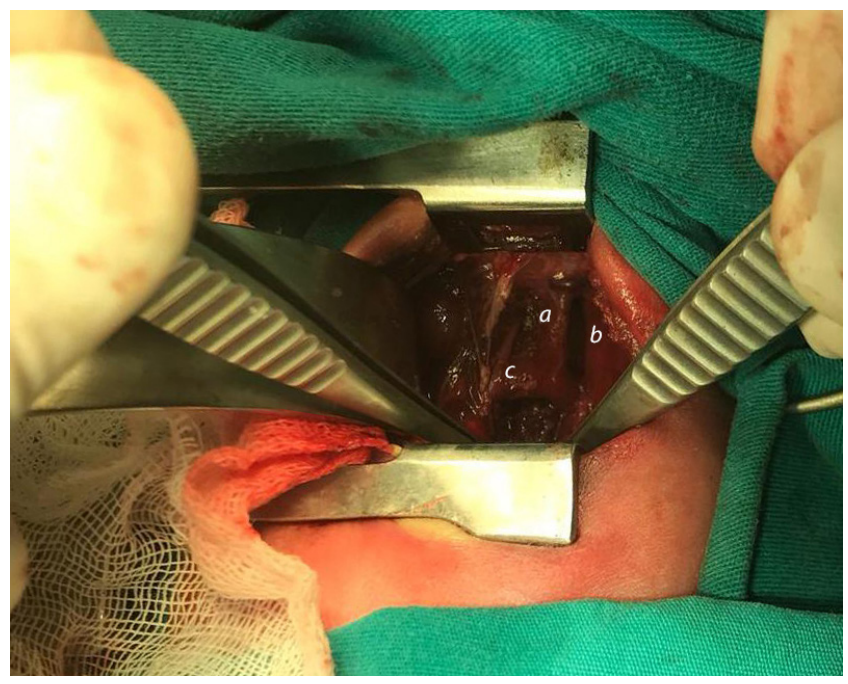

Figure 2: Intraoperative image (a: proximal atresia, $b$ : trachea, $c$ : distal fistula). 


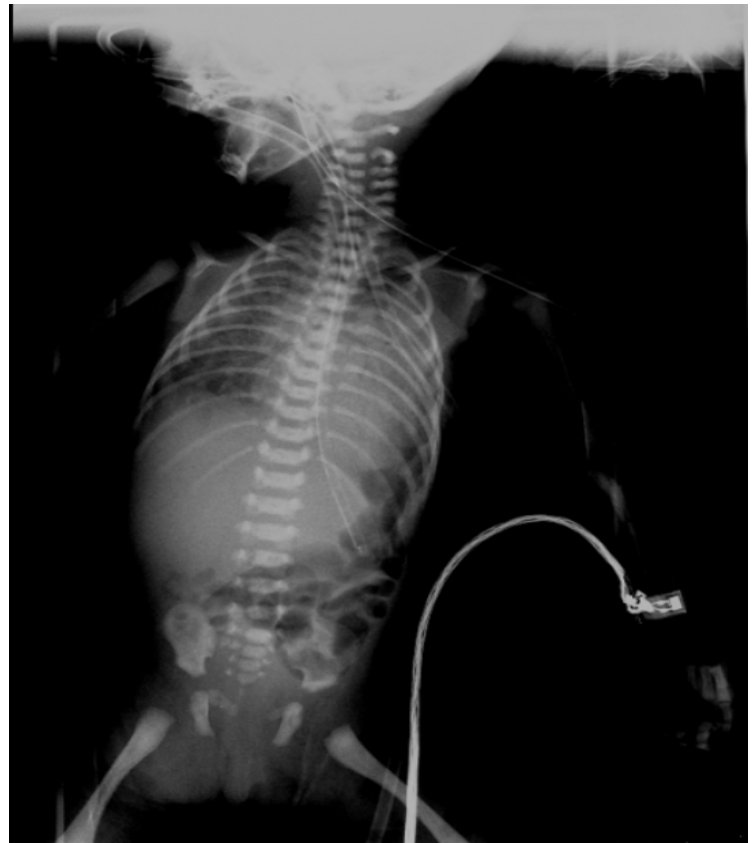

Figure 3: Postoperative plain X-Ray film (No radiocontrast liquid accumulation is seen).

\section{DISCUSSION}

The most common newborn esophageal malformation is esophageal atresia (9). It can be diagnosed in a prenatal period, at delivery or in a postnatal period by the responsible physicians. The complete swallowing process can be visualized by video-fluoroscopy and a definitive diagnosis can be achieved (9). Surgical treatment is essential for patients suffering from EA. New methods are being developed for its surgical treatment. Specifically, for short-gap EA, type-C according to Gross (4), primer anastomosis of distal and proximal parts is the most used surgical method. In this method, the surgeon attaches distal and proximal parts by suturing them together and forms a proper esophagus (8). This open or thoracoscopic chest surgery has life-threatening complications and causes a lifelong-carried scar (8). The major lifelong-carried complication of this operation is esophageal dysmotility and its related problems like GER. In the post-operative period, 34\% of patients suffer from GER caused by esophageal dysmotility (8). Patients may continue to have problems with these basic reflexes and may need physical therapy even when they are treated well surgically. 30\% of children with EA undergoes fundoplication for GER treatment (10). A study made by Menzies et al. (11) showed that failure to thrive is seen at patients who had fundoplication. In addition to failure to thrive, inste- ad of fundoplication, studies showed that transpyloric feeding is more beneficial for the patient in different aspects like growth and reducing the effects of reflux $(12,13)$.

The patient in our case received physical therapy and was followed-up for ten months after the surgery. Although we have seen a full recovery and the patient was able to swallow food at the end of the treatment the diagnosis could be achieved during the prenatal period and the surgery and the required follow-up therapy could be planned more easily.

Today, a new method developed by Morrow et al. (14) is aiming to decrease the risk of long-term complications of the operation. In this method, the physician uses magnets to reattach two parts of the esophagus. At patients with shorter than 4 centimeters long gap, this method has been successful and approved by the United States Food and Drug Administration (14). Morrow's method does not require a primer anastomosis to reattach two parts of the esophagus and maintain a proper integration naturally. This natural integration of proximal and distal ends minimalizes the risk of damaging nerves, which are innervating the esophagus and correlatively minimalizing the risk of esophageal dysmotility. This new method is being tried by many physicians and their experiences are being reported (14). In the future, it can be a safer option. But today, primer anastomosis is still the most common surgical method for EA.

As a conclusion, esophageal atresia with distal tracheoesophageal fistula is not an uncommon malformation. The patients can have problems with swallowing in their infancy even when they are treated surgically in the neonatal period. Therefore patients need to be followed-up and may need a referral to a physical medicine and rehabilitation specialist for appropriate postoperative treatment.

\section{Ethics Committee Approval: N/A}

Informed Consent: Written informed consent was obtained from the patient's family for this study.

Conflict of Interest: The authors declared no conflict of interest.

Author contributions: Concept: AUM, Mİ Supervision: AUM,MI Resources: AUM Materials: AUM, OK Data collection and/or processing: AUM Analysis and/or Interpretation: AUM, OK Literature Search: AUM Writing Manuscript: AUM Critical Review: MI

Financial disclosure: The authors declared that this study received no financial support

Editor-in-chief's Note: One of the authors of this article, Arda Ulaş Mutlu is a member of the editorial board of Turkish Medical Student Journal. However, he did not take place in any stage on the editorial decision of the manuscript. The editors who evaluated this manuscript are from other institutions. 


\section{REFERENCES}

1. Conforti A, Morini F, Bagolan P. Difficult esophageal atresia: trick and treat. Semin Pediatr Surg 2014;23(5):261-9.

2. Smith N. Oesophageal atresia and tracheo-oesophageal fistula. Early Hum Dev 2014;90(12):947-50.

3. Sfeir R, Michaud L, Sharma D et al. National esophageal atresia register. Eur J Pediatr Surg 2015;25(6):497-9.

4. Gross RE. The surgery of infancy and childhood. 1st ed. Philadelphia: WB Saunders; 1953.

5. Macchini F, Parente G, Morandi A et al. Classification of esophageal strictures following esophageal atresia repair. Eur J Pediatr Surg 2018;28(3):243-9.

6. Larsen WJ. Embryonic folding. In: Larsen JW, ed. Essentials of Human Embryology. New York, NY: Churchill Livingstone; 1998:143-4. 7. Connor MJ, Springford LR, Kapetanakis VV et al. Esophageal atresia and transitional care-step 1: a systematic review and meta-analysis of the literature to define the prevalence of chronic long-term problems. Am J Surg 2015;209(4):747-59.

8. Sistonen SJ, Koivusalo A, Nieminen U et al. Esophageal morbidity and function in adults with repaired esophageal atresia with tracheoesophageal fistula a population-based long-term follow-up. Ann Surg 2010;25:1167-73.

9. Scheeren B, Maciel AC, Barros SG. Videofluoroscopic swallowing study: esophageal alterations in patients with dysphagia. Arquivos de Gastroenterologia 2014;51:221-5.

10. Kawahara H, Kubota A, Hasegawa T et al. Lack of distal esophageal contractions is a key determinant of gastroesophageal reflux disease after repair of esophageal atresia. J Pediatr Surg 2007;42:2017-

21.

11. Menzies J, Hughes J, Leach S et al. Prevalence of malnutrition and feeding difficulties in children with esophageal atresia. J Pediatr Gastroenterol Nutr 2017;64(4):100-5.

12. Rosen R, Hart K, Warlaumont M. Incidence of gastroesophageal reflux during transpyloric feeds. J Pediatr Gastroenterol Nutr 2011;52(5):532-5.

13. Rosen R, Levine P, Lewis J et al. Reflux events detected by $\mathrm{pH}-$ MII do not determine fundoplication outcome. J Pediatr Gastroenterol Nutr 2010;50(3):251-5.

14. Morrow T. Wilson-cook's flourish device uses magnets to fix pediatric esophageal atresia. Manag Care 2017;26(9):32-3. 